\title{
ON THE EQUILIBRIUM OF CHEMICAL SYSTEMS
}

\author{
BY PAUL SAUREL
}

\section{INTRODUCTION}

Among the many theorems which Gibbs has given in his famous memoir: On the Equilibrium of Heterogeneous Substances, ${ }^{2}$ one of the most important for physical chemistry, if indeed not the most important, is the theorem which goes by the name of the phase rule. This theorem can be stated as follows: If a heterogeneous system consisting of $r$ homogeneous pliases formed by means of $n$ independent components be in equilibrium, there exist between the temperature, the pressure and the $n r$ concentrations, $n r+r-n$ equations, so that, if $n-r+2$ of these variables be given the values of the others will be fixed.

This theorem allows us to classify chemical systems according to the value of the number $n-r+2$, which has been called the variance of the system. In accordance. with 'Trevor's nomenclature a system is said to be invariant, univariant, bivariant, trivariant, etc., according as the variance is equal to zero, to one, to two, to three, etc.; when the variance exceeds two the system is said to be multivariant.

Gibbs' theorem, however, does not furnish all the information which is necessary to characterize the state of equilibrium of a heterogeneous substance. Duhem ${ }^{3}$ has completed it by

1 This article is a translation of the more important parts of a thesis Sur l'équilibre des systèmes chimiques, which was presented to the Faculté des Sciences of Bordeaux in April, Inoo.

2 Transactions of the Connecticut Academy of Arts and Sciences, 3, Part I, I876; 3, Part II, I878. J. Willard Gibbs. Thermodynamische Studien. Translated by W. Ostwald, Leipzig, 1892. J, W. Gibbs, Équilibre des systèmes chimiques. Translated by H. Le Châtelier, Paris, 1899.

' Jour. Phys. Chem, 2, 38, 103 (1898). Traité elémentaire de' Mécanique chimique, 4, $294,35 \pi$. 
showing that if the equations which connect the masses of the phases with the masses of the independent components and the volume of the system be taken into account, the equilibrium of a chemical system can be completely discussed. The results which are obtained in this way, when the masses of the independent components are given, are the following:

An invariant system can be in equilibrium only at a definite temperature, under a definite pressure and for definite values of the concentrations. At the equilibrium temperature and under the equilibrium pressure, the system admits of a continuous series of states of equilibrium in which the masses of the phases vary while the concentrations remain constant. The state of equilibrium of the system is completely determined if the values of two other variables be given, for example, the masses of two of the phases or the mass of one phase and the volume of the system, or again the volume and the entropy of the system.

A univariant system can be in equilibrium at an infinity of temperatures, but to each temperature there corresponds an equilibrium pressure and equilibrium concentrations. At a given temperature and under the equilibrium pressure which corresponds to that temperature, the system admits of a continuous series of states of equilibrium in which the masses of the phases vary while the concentrations remain constant. The state of equilibrium is completely determined if the value of one other variable be given, for example the mass of one phase or the volume of the system.

A bivariant or multivariant system can be in equilibrium at an infinity of temperatures and at each of these temperatures under an infinity of pressures. At a given temperature and under a given pressure the concentrations and the masses of the phases are determinate; the state of equilibrium is accordingly completely determined.

Duhem has gone further and has shown that, if the masses of the independent components of a bivariant system be suitably chosen, the system behaves like a univariant system, that is to say, to a given temperature there correspond an equilibrium 
pressure and equilibrium concentrations, and, at the given temperature and under the corresponding pressure, the system admits of a continuous series of states of equilibrium in which the masses of the phases vary while the concentrations remain constant. Duhem has given the name of indifferent point of a bivariant system to a state of equilibrium in which the system possesses the above mentioned properties of a univariant system.

It is natural to ask if the notion of indifferent point can be extended to multivariant systems. We have found that such an extension is indeed possible; if the masses of the independent components be suitably chosen, the multivariant system behaves like a univariant system. This result completes the discussion of the equilibrium of a heterogeneous system.

The theorems which we have just recalled relate to the equilibrium of a system; there is another series of important theorems which relate to the displacement of this equilibrium. In the first place there is a theorem which applies to univariant systems, viz.: Clapeyron's equation, which connects the simultaneous variations of the temperature and the equilibrium pressure. Gibbs ${ }^{x}$ has shown that at an indifferent point of a bivariant system, formed by means of two components, the same equation holds; it is thus natural to ask if the application of this equation can be extended to the indifferent points of bivariant or multivariant systems formed by means of any number of independent components. We have found that such an extension is indeed possible.

For bivariant systems Gibbs ${ }^{2}$ has given the following two theorems, of which the first has been independently rediscovered by Konowalow: ${ }^{3}$

If we consider, at a constant temperature, a bivariant system whose mass can be varied, the pressure of the system at the indifferent point which corresponds to the given temperature is, in general, a maximum or a minimum of the pressures possible

${ }^{1}$ On the Equilibrium of Heterogeneous Substances, p. 15.;.

2 Ibid. p. I 55 .

${ }^{3}$ Wied. Ann. 14, 48 (1881). 
at the given temperature. Similarly, if we consider a bivariant system under a constant pressure, the temperature of the system at the indifferent point which corresponds to the given pressure is, in general, a maximum or a minimum of the temperatures possible under the given pressure.

Gibbs' demonstration is capable of immediate extension to the indifferent points of multivariant systems.

There remain to be mentioned two other important theorems which apply to bivariant and to multivariant systems, viz.: Le Châtelier's theorem on the displacement of equilibrium by variation of pressure and van 't Hoff's theorem on the displacement of equilibrium by variation of temperature. ${ }^{2}$ The first general demonstration of these theorems is due to Duhem. ${ }^{3}$

To establish the various theorems on the displacement of equilibrium which we have just recalled, demonstrations have been given which can not be easily connected; accordingly, it seemed desirable to deduce them all from a single formula. Planck $^{4}$ has given a general formula for the displacement of equilibrium, and he has made use of it to deduce Clapeyron's formula for univariant systems. Starting with Planck's formula we have been able to establish without difficulty the various theorems on the displacement of equilibrium, viz.: Clapeyron's equation for univariant systems and for the indifferent points of bivariant and multivariant systems, the theorems of Gibbs and Konowalow on indifferent points and finally the theorems of Le Châtelier and van 't Hoff.

We must now indicate the plan of this article.

In the first chapter we begin by recalling the criterium of equilibrium which is used in what follows. This criterium is the one that makes use of Gibbs' ' -function. Duhem has given to this function the name total thermodynamic potential under constant pressure, and he has shown its great importance and usefulness in the study of the equilibrium of chemical systems.

\footnotetext{
${ }^{1}$ Compt. rend. 99, 786 (1884).

2 Études de Dynamique chimique, p. I6I; Amsterdam, I884.

"Annales de la Faculté de Toulouse, 4, N, (1890).

*Vorlesungen über Thermodynamik, p. I76; Leipzig, I 897 .
} 
We then give a demonstration of the phase rule which, in substance, is identical with the demonstrations of Gibbs and of Duhem, but which, in form, contains some simplifications.

In the second chapter we establish the conditions for the existence of an indifferent point in a bivariant system and in a multivariant system. These conditions enable us to give demonstrations of Clapeyron's theorems and of the theorems of Gibbs and Konowalow.

Finally, in the third chapter, starting with Planck's equation, we deduce the various theorems on the displacement of equilibrium : the theorems of Clapeyron, of Gibbs and Konowalow, of Le Châtelier, and of van't Hoff.

The object we have had in view has been to put the theory of the equilibrium of heterogeneous systems into as compact and as simple a form as possible and, at the same time, to fill in some gaps in the theory.

This paper was written at the University of Bordeaux in the spring of Igoo, and I wish to express here my gratitude to Professor P. Duhem of that University, for his unfailing kindness to me, and for the interest with which he followed the progress of $11 y$ work.

\section{CHAPTER I.}

\section{THE EQUILIBRIUM OF HETEROGENEOUS SYSTEMS}

\section{;. The Phase Rule}

Let us consider a heterogeneous system of $r$ phases formed by means of $n$ independent components whose masses are $\mathfrak{M}_{1}, \mathfrak{N}_{2}, \ldots, \mathfrak{N}_{n}$. By independent components of the system we mean substances, simple or otherwise, such that every system similar to the given system can be formed by taking suitable masses of these substances. Thus, for example, we say that the system which is formed by the dissociation of a mass of calcium carbonate into quicklime and carbon dioxide, is formed by 
means of the two independent components $\mathrm{CaO}$ and $\mathrm{CO}_{2}$. For, although the system considered has been formed from a single mass of $\mathrm{CaCO}_{3}$, we can obtain similar systems by adding an excess of $\mathrm{CaO}$ or of $\mathrm{CO}_{2}$.

If we denote by $\mathrm{E}, \mathrm{H}$, and $\mathrm{V}$ the energy, the entropy and the volume of the heterogeneous system, and by $T$ and $\Pi$ its temperature and its pressure, the total thermodynamic potential $\Phi$ of the system is defined by the equation.

$$
\Phi=\mathrm{E}-\mathrm{TH}+\Pi \mathrm{V} .
$$

Similarly, if we denote by $\mathrm{E}_{i}, \mathrm{H}_{i}$ and $\mathrm{V}_{i}$ the energy, the entropy and the volume of the $i$-th phase, the total thermodyaamic potential $\Phi_{i}$ of that phase is defined by the equation

$$
\Phi=\mathrm{E}_{i}-\mathrm{TH}_{i}+\Pi \mathrm{V}_{i} .
$$

Now it is evident that

$$
\mathrm{V}=\sum_{i=1}^{r} \mathrm{~V}_{i}
$$

if then we admit that

$$
\mathrm{E}=\sum_{i=1} \mathrm{E}_{i,} \quad \mathrm{H}=\sum_{i=\mathrm{I}}^{r} \mathrm{H}_{i},
$$

it follows that

$$
\Phi=\sum_{i=1}^{r} \Phi_{i} .
$$

From the definition of $\Phi$ it can be shown that it is a homogeneous function of the first degree in the masses of the independent components. If, moreover, we admit that $\Phi$ is a function of the temperature and the pressure, it can be shown that when the system is in equilibrium

$$
\frac{\partial \Phi}{\partial T}=-\mathrm{H}, \quad \frac{\partial \Phi}{\partial \Pi}=\mathrm{V} .
$$

In like nanner, we shall admit that the total thermodynamic potential of the $i$-th phase is a function of the temperature 
and the pressure, and it can then be shown that when the phase is in equilibrium

$$
\frac{\partial \Phi_{i}}{\partial I^{1}}=-\mathrm{H}_{i}, \quad \frac{\partial \Phi}{\partial \Pi}=V_{i}
$$

Moreover, if we denote by $\mathrm{M}_{i j}$ the mass of the $j$-th component which enters into the $i$-th phase, $\Phi_{i}$ will be a homogeneous function of the first degree in $M_{i 1}, M_{i 2}, \ldots, M_{i n}$. We can therefore write

$$
\Phi_{i}=\sum_{j=\mathrm{I}}^{n} \frac{\partial \Phi_{i}}{\partial \mathrm{Ni}_{i j}} \mathrm{M}_{i j} .
$$

We shall also write the formula which gives the variation of the total potential $\Phi_{i}$, when the phase undergoes a virtual change which leaves the temperature and the pressure unaltered. The variation in question is given by the equation

$$
\delta \Phi_{i}=\sum_{j=\mathrm{t}}^{n} \frac{\partial \Phi_{i}}{\partial \mathrm{M}_{i j}} \delta \mathrm{M}_{i j} .
$$

The partial derivatives which appear in equations 8 and 9 are homogeneous functions of the degree zero in $\mathrm{M}_{i \mathrm{I}}, \mathrm{M}_{i_{2}}, \ldots$, $\mathrm{M}_{i n}$; in other words, they are functions of the ratios of these masses. If, then, we denote by $m_{i j}$ the mass of the $j$-th component which enters into the unit of mass of the $i$-th phase, the derivatives in question will be functions of $m_{i 1}, m_{i 2}, \ldots, m_{i n}, \mathrm{~T}, \Pi$.

If the $j$-th component of the system is one of the independent components of the $i$-th phase, we shall write

$$
\mathrm{F}_{i j}=\frac{\partial \Phi_{i}}{\partial \mathrm{M}_{i j}}
$$

If the independent components of the system are not independent components of the $i$-th phase, the masses $M_{i 1}, M_{i 2}, \ldots$, $M_{i n}$ satisfy a certain number of conditions of the form

$$
\mathrm{M}_{i j}=\lambda \mathrm{M}_{i k,}, \quad \mathrm{M}_{i j}==\mu \mathrm{M}_{i \ell}, \quad \cdots,
$$

in which $\lambda, \mu, \ldots$, are constants. As a consequence of these equations we shall have 


$$
\delta \mathrm{M}_{i j}=\lambda \delta \mathrm{M}_{i k,}, \quad \delta \mathrm{M}_{i j}=\mu \delta \mathrm{M}_{i l}, \quad \ldots .
$$

But then, in the expressions for $\Phi_{i}$ and $\delta \Phi_{i}$, we may replace the terms

$$
\begin{aligned}
& \frac{\partial \Phi_{i}}{\partial \mathrm{M}_{i j}} \mathrm{M}_{i j}+\frac{\partial \Phi_{i}}{\partial \mathrm{M}_{i k}} \cdot \mathrm{M}_{i k}+\frac{\partial \Phi_{i}}{\partial \mathrm{M}_{i l}} \mathrm{M}_{i l}+\cdots \\
& \frac{\partial \Phi_{i}}{\partial \mathrm{M}_{i j}} \delta \mathrm{M}_{i j}+\frac{\partial \Phi_{i}}{\partial \mathrm{M}_{i k}} \delta \mathrm{M}_{i z}+\frac{\partial \Phi_{i}}{\partial \mathrm{M}_{i l}} \delta \mathrm{M}_{i l}+\cdots
\end{aligned}
$$

by the terms

$$
\begin{aligned}
\left(\frac{\partial \Phi_{i}}{\partial \mathrm{M}_{i j}}+f+g+\ldots\right) \mathrm{M}_{i j}+\left(\frac{\partial \Phi_{i}}{\partial \mathrm{M}_{i, k}}-\lambda f\right) \mathrm{M}_{i k} & \\
& +\left(\frac{\partial \Phi_{i}}{\partial \mathrm{M}_{i l}}-\mu g\right) \mathrm{M}_{i l}+\ldots \\
\left(\frac{\partial \Phi_{i}}{\partial \mathrm{M}_{i j}}+f+g+\ldots\right) \delta \mathrm{M}_{i j}+\left(\frac{\partial \Phi_{i}}{\partial \mathrm{M}_{i k}}-\lambda f\right) \delta \mathrm{M}_{i k} & -\left(\frac{\partial \Phi_{i}}{\partial \mathrm{M}_{i l}}-\mu g\right) \delta \mathrm{M}_{i l}+\ldots \\
& +\cdots
\end{aligned}
$$

$f, g, \ldots$ being arbitrary functions. We shall then write

$$
\begin{aligned}
& \mathrm{F}_{i j}=\frac{\partial \Phi_{i}}{\partial M_{i j}}+f+g+\cdots, \\
& \mathrm{F}_{i k}=\frac{\partial \Phi_{i}}{\partial M_{i k}}-\lambda f, \\
& \mathrm{~F}_{i l}=\frac{\partial \Phi_{i}}{\partial \mathrm{M}_{i l}}-\mu g,
\end{aligned}
$$

$f, \mathrm{~g}, \ldots$ being arbitrary functions whose number is the same as that of the equations of condition II.

If the $j$-th component does not enter into the $i$-th phase, that is to say if

$$
\begin{aligned}
\mathrm{M}_{i j} & =0, \\
\delta \mathrm{M}_{i} & =0,
\end{aligned}
$$


the derivative $\frac{\partial \Phi_{i}}{\partial \mathrm{M}_{i j}}$ will be equal to zero and will disappear from equations 8 and 9. In that case we shall put

$$
\mathrm{F}_{z j}=0 \text {. }
$$

Then instead of equations 8 and 9 we may write

$$
\begin{gathered}
\Phi_{i}=\sum_{j=\mathrm{x}}^{n} \mathrm{~F}_{i j} \mathrm{M}_{i j}, \\
\delta \Phi_{2}=\sum_{j=1}^{n} \mathrm{~F}_{i j} \delta \mathrm{M}_{i j} .
\end{gathered}
$$

The thermodynamic potential of the system of $r$ phases is accordingly given by the equation

$$
\Phi=\sum_{i=\mathrm{I}}^{r} \sum_{j=\mathrm{I}}^{n} \mathrm{~F}_{i j} \mathrm{M}_{i j}
$$

and the variation of this potential during a virtual change which leaves the temperature and the pressure unaltered is given by the equation

$$
\delta \Phi=\sum_{i=\mathrm{r}}^{r} \sum_{j=1}^{n} \mathrm{~F}_{i j} \delta \mathrm{M}_{i j}
$$

Let us suppose that, for the system of $r$ phases, there are $p$ conditions of the form I 3 and $q$ conditions of the form II. Then $p$ of the quantities $F_{i j}$ are identically equal to zero and disappear from equations $I 7$ and $I 8$. In the quantities $F_{i j}$ which remain there appear $q$ arbitrary functions which we may choose in any way that is convenient.

The variations $\delta \mathrm{M}_{i j}$ which appear in equation $\mathrm{I} 8$ are not all independent. In the first place, the definition of the masses $M_{i j}$ shows that they satisfy the $n$ equations

$$
\sum_{i=1}^{r} \mathrm{M}_{i j}=\mathfrak{9 K}_{j}, \quad j=\mathrm{I}, 2, \ldots, n .
$$


In a change which leaves the total mass of each component unaltered, the variations $\delta M_{i j}$ must accordingly satisfy the $n$ equations

$$
\sum_{i=1}^{r} \delta \mathrm{M}_{i j}=0, \quad j=\mathrm{I}, 2, \ldots, n .
$$

Moreover, these variations must satisfy $p$ conditions of the form I 4 ,

$$
\delta \mathrm{M}_{i j}=\mathrm{O},
$$

and $q$ conditions of the form I 2

$$
\delta \mathrm{M}_{i j}=\lambda \delta \mathrm{M}_{i k}
$$

Among the $n r$ quantities $\delta M_{i j}$, there are thus only $n r-n-p-q$ which are independent.

These preliminaries being established we can now apply the criterium of equilibrium. The system will be in equilibrium if, for every virtual change which leaves the temperature, the pressure and the masses of the components unaltered, we have

$$
\delta \Phi \geq 0 \text {. }
$$

If we suppose that no new phase can be formed, and that each phase actually contains a mass of each of its components, all the virtual changes will be revertible, and the preceding condition will reduce to

$$
\delta \Phi=\text { o. }
$$

Thus the system will be in equilibrium if

$$
\sum_{i=1}^{r} \sum_{j=1}^{n} \mathrm{~F}_{i j} \delta \mathrm{M}_{i j}=0
$$

for all the variations $\delta \mathrm{M}_{i j}$ which satisfy equations $20,2 \mathrm{I}$ and 22 .

If we multiply equations 20 by the arbitrary quantities $F_{1}$, $\mathrm{F}_{2}, \ldots, \mathrm{F}_{n}$, and if we subtract the results from equation 23 , the condition of equilibrium will become

$$
\sum_{i=\mathrm{I}}^{r} \sum_{j=\mathrm{I}}^{n}\left(\mathrm{~F}_{i j}-\mathrm{F}_{j}\right) \delta \mathrm{M}_{i j}=0 .
$$


In this equation there are $p$ terms which are identically equal to zero, namely, the terms which correspond to the $p$ equations 2I. Let us take $\mathrm{F}_{j}$ equal to one of the functions $\mathrm{F}_{\mathrm{I} j}$, $F_{2 j}, \ldots, F_{r j}$, which is not identically equal to zero; on account of this choice $n$ more terms disappear from the equation. Finally, let us choose the $q$ arbitrary functions $f, g, \ldots$, which appear in certain of the functions $F_{i j}$, in such a way that the coefficients of the $q$ variations, such as $\delta \mathrm{M}_{i k}$ of equation 22, shall be identically equal to zero. There will then remain in equation 24 only the terms containing the $n r-n-p-q$ independent variations; we must then equate the coefficients of these variations to zero.

The conditions which. we obtain in this way may be written in the form

$$
\mathrm{F}_{x j},=\mathrm{F}_{2 j}=\cdots=\mathrm{F}_{x j}, \quad j=\mathrm{I}, 2, \ldots, n,
$$

if we agree not to write the $p$ functions $F_{i j}$ which are identically equal to zero. Because of this convention, the number of equations 25 is only $n(r-\mathrm{r})-p$. But we must observe that the choice which we made of the $q$ arbitrary functions $f, g, \ldots$ which appeared in certain of the functions $\mathrm{F}_{i j}$, is such that $q$ of the equations 25 are identities. The number of conditions 25 is thus only $n r-n-p-q$.

Equations 25 thus furnish $n r-n-p-q$ relations between the $n r+2$ quantities $m_{i j}$, T, II. To these equations we must add the $r$ equations

$$
\sum_{j=1}^{n} m_{i j}=1, \quad i==1,2, \ldots, r
$$

which follow at once from the definition of the quantities $m_{i j}$, the $p$ equations

$$
m_{i j}=0 \text {, }
$$

which correspond to the $p$ equations I 3 , and the $q$ equations

$$
m_{i j}=\lambda m_{i k}
$$

which correspond to the $q$ equations Ir. Thus, between the 
temperature $T$, the pressure $\Pi$ and the $n r$ concentrations $m_{i j}$, there exist $n r-n-p-q \div r+p+q$ relations; there are then only $n-r+2$ of these variables which are independent. This theorem of $\mathrm{Gibbs}^{\mathrm{x}}$ is known as the phase rule.

\section{\%2. Classification of Heterogeneous Systems}

As Duhem has shown, ${ }^{2}$ we can complete the study of the equilibrium of a heterogenous system, if we take into account the relations which exist between the masses of the phases and the masses of the components.

I,et us denote by $\mathrm{M}_{i}$ the mass of the $i$-th phase; then

$$
\mathrm{M}_{i j}=m_{i j} \mathrm{M}_{i} \text {, }
$$

and equations Ig become

$$
\sum_{i=\mathrm{r}}^{r} m_{i j} \mathrm{M}_{i}=\mathfrak{N}_{j}, \quad j=\mathrm{I}, 2, \ldots, n
$$

Moreover, if we denote by $v_{i}$ the volume of the unit of mass of the $i$-th phase, we shall have

$$
\mathrm{V}_{i}=v_{i} \mathrm{M}_{i}
$$

and

$$
\sum_{i=1}^{r} v_{i} \mathrm{M}_{i}=\mathrm{V}
$$

When the system is in equilibrium, the specific volume $z_{i}$ can be obtained from the thermodynamic potential of the unit of mass of the $i$-th phase by means of a formula similar to the one by which the volume of the system is obtained from the thermodynamic potential of the system; this specific volume is thus a function of $m_{i 1}, m_{i 2}, \ldots, m_{i n}, T, \Pi$.

The number $n-r+2$ is called the variance of the system. If $r=n+2$, the variance is equal to zero, and the system is called an invariant system. In this case, the variables $T, \Pi, m_{i j}$

"On the Equilibrium of Heterogeneous Substances, p. 152.

2 Jour. Phys. Chem. 2, 38, I03 (1898). Traité élémentaire de Mécanique chimique, 4, 294, 35 I. 
have determinate values. Let us suppose that the masses $\mathfrak{N}_{j}$ are given; then if we choose arbitrarily the masses of two of the phases, the $n$ equations 30 will determine the masses of the $r-2$ remaining phases. Thus, if the masses of the components of an invariant system are given, the equilibrium of the system is indifferent.

If we know not only the masses of the components, but also the volume $\mathrm{V}$ of the system, then we can choose arbitrarily the mass of only one phase; the $n+\mathrm{I}$ equations $3^{\circ}$ and 32 will determine the masses of the $r-I$ remaining phases. The equilibrium of the system is still indifferent.

If $r=n+I$, the variance of the system is equal to one and the system is called a univariant system. In this case we may choose arbitrarily one of the variables $\mathrm{T}, \Pi, m_{i j}$; the others are then determinate. Let us suppose that the masses $\mathfrak{O K}_{j}$ are given; then, if we choose arbitrarily the mass of one of the phases, the $n$ equations 30 will determine the masses of the remaining $r-$ I phases. Thus, the masses of the components being given, if we know also the temperature or the pressure, the equilibrium of the univariant system is indifferent.

If, in addition to the masses of the components, the volume of the system be given, the $n-1$ I equations 30 and 32 will determine the masses of the $r$ phases. Thus, the masses of the components being given, if we know also the temperature and the volume or the pressure and the volume, the state of equilibrium of the mivariant system is determined.

If $r=n$, the variance of the system is equal to two, and the system is called a bivariant system. In this case, we may choose arbitrarily two of the variables $\mathrm{T}, \Pi, m_{i j}$; the others are then determinate. If the masses $\mathscr{N}$; are given, the $n$ equations 30 will determine the masses of the $r$ phases. Thus, the masses of the components being given, if we know also the temperature and the pressure, the state of equilibrium of the bivariant system is determined.

Suppose that we know not only the masses of the components but also the volume of the system. The $n$ equations 30 
determine the masses of the $r$ phases in terms of the concentrations $m_{i j}$. If we substitute these values of the masses $M_{i}$ in equation 32 , we shall have a new relation between the variables T, $\Pi, m_{i}$. There is then but one of these variables which can be chosen arbitrarily. Thus, the masses of the components being given, the state of equilibrium of the bivariant system will be determined if the temperature and the volume or the pressure and the volume be given. The state of equilibrium of a bivariant system is thus, in general, determined if we know the masses of the components and two of the three variables: temperature, pressure and volume.

When the variance exceeds two, the system is called a multivariant system. If $r=n-p$, the variance of the system is equal to $p+2$. In this case $p+2$ of the variables $m_{i j}$, T, $\Pi$ are independent. If the masses $\mathfrak{N H}_{j}$ are given, $n-p$ of the equations 30 will determine the masses of the $r$ phases in terms of the concentrations $m_{i j}$. If we substitute these values of the masses in the $p$ remaining equation 30 , we shall obtain $p$ new relations between the concentrations. We can then choose arbitrarily only two of the quantities $T, \Pi, m_{i j}$. Thus the state of equilibrium of a multivariant system is determined if the masses of the components, the temperature and the pressure are given.

Suppose that we know not only the masses of the components, but also the volume of the multivariant system. From $n-p$ of the equations 30 we can determine the masses of the $r$ phases in terms of the concentrations. If we substitute these values of the masses in the $p$ remaining equations 30 and in equation 32 , we shall obtain $p+$ I new relations between the variables $T, \Pi, m_{i j}$. We can thus choose arbitrarily only one of these quantities. Thus the state of equilibrium of a multivariant system is determined if, in addition to the masses of the components, the temperature and the volume or the pressure and the volume are given. The state of equilibrium is thus, in general, determined if we know the masses of the components and two of the three variables: temperature, pressure and volume.

From the preceding discussion it follows that the state of 
equilibrium of any system is, in general, determined if we know the masses of the components and the values of two other variables. In an invariant system we can take, as independent variables, the masses of two phases or the volume of the system and the mass of one phase. In a univariant system we can choose the temperature or the pressure as one variable, and the volume of the system or the mass of one phase as the second variable. Finally, in a bivariant or multivariant systenn we can take any two of the three variables: temperature, pressure and volume.

CHAPTER II. INDIFFERENT POINTS

\section{\&I. Indifferent Points of Bivariant Systems}

In the last chapter we have shown that for a bivariant or multivariant system we may choose arbitrarily the temperature and the pressure and that then, if the masses of the components are given, the state of equilibrium of the system is, in general, determinate. There exist however exceptional states for which this proposition is no longer true and in which the bivariant or multivariant system behaves like a univariant system, that is to say, the temperature and the equilibrium pressure are connected by an equation, and, the masses of the components being given, at a given temperature or under a given pressure, the system admits of a continuous series of states of equilibrium. We shall give the name of indifferent point of a bivariant or multivariant system to a state of equilibrium of the system in which it possesses the properties of a univariant system which we have just recalled.

Consider a bivariant system and write the equations 30 which correspond to that case: 


$$
\sum_{i=1}^{n} m_{i j} \mathrm{M}_{i}=9 \mathbb{M}_{j,} \quad j=1,2, \ldots, n
$$

Let us write

$$
\begin{array}{cccc}
m_{11}, & m_{21}, & \cdots, & m_{n \mathrm{I}} \\
m_{12}, & m_{22}, & \cdots, & m_{n 2} \\
\vdots & \vdots & & \vdots \\
m_{1 n}, & m_{2 n}, & \cdots, & m_{n n}
\end{array}
$$

and let us denote by $A_{i j}$ the minor of this determinant which corresponds to the element $m_{i j}$. Then multiplying equations 33 by $A_{i 1}, A_{i 2}, \ldots, A_{i n}$ and adding, we obtain the following equations which must be satisfied by all the values of the quantities $\mathrm{M}_{i}$ which satisfy equations 33 :

$$
\mathrm{M}_{i} \mathrm{~A}=\sum_{i=1}^{n} \mathfrak{N}_{j} \mathrm{~A}_{i j}, \quad, \quad i=\mathrm{I}, 2, \cdots, n,
$$

As is well known, these equations give, in general, the values of the quantities $M_{i}$ which satisfy equations 33 . But if

$$
\mathrm{A}=\mathrm{o} \text {, }
$$

this is no longer the case. However, as the equations 35 must be satisfied by all the values $\mathrm{M}_{i}$ which satisfy equations 33 , if we suppose that there are finite values of the masses $\mathrm{M}_{i}$ which satisfy these equations, we must also have

$$
\sum_{j=1}^{n} \mathfrak{N K}_{j} \mathrm{~A}_{i j}=0, \quad i=1,2, \cdots, n .
$$

The masses $\mathfrak{M K}_{j}$ must accordingly satisfy the $n$ homogeneous equations 37 . The necessary and sufficient condition for this is that the determinant of these equations should be equal to zero. But this condition is satisfied, for

$$
\left|\begin{array}{cccc}
\mathrm{A}_{11}, & \mathrm{~A}_{12}, & \ldots, & \mathrm{A}_{I n} \\
\mathrm{~A}_{21}, & \mathrm{~A}_{22}, & \ldots, & \mathrm{A}_{2 n} \\
\vdots & \vdots & & \vdots \\
\mathrm{A}_{n \mathrm{I}}, & \mathrm{A}_{n 2}, & \ldots, & \mathrm{A}_{n n}
\end{array}\right|=\mathrm{A}^{n-1}=0
$$


Suppose then that the condition 36 is satisfied and that the masses $\mathfrak{N} \tilde{\hat{j}}_{j}$ have been so chosen as to satsify equations 37 . This choice can be made in an infinite number of ways since equations 37 determine only the ratios of the quantities $\mathscr{N}_{j}$. Under these conditions, if we choose arbitrarily the mass of one of the phases, $M_{\mathrm{r}}$ for example, the other masses will be determined by equations 33. To show this, let us, for the moment, disregard the first of equations 33 and let us write the others in the form

$$
\sum_{i=2}^{n} m_{i j} \mathrm{M}_{i}=\mathfrak{N}_{j}-m_{j j} \mathrm{M}_{1}, \quad j=2,3, \ldots, n .
$$

Let us write

$$
\left|\begin{array}{cccc}
m_{22}, & m_{32}, & \ldots, & m_{n 2} \\
m_{23}, & m_{33}, & \ldots, & m_{n 3} \\
\vdots & \vdots & & \vdots \\
m_{2 n}, & m_{3 n}, & \ldots, & m_{n n}
\end{array}\right|=\mathrm{B}
$$

and let us denote by $\mathrm{B}_{i j}$ the minor of this determinant which corresponds to the element $m_{i j}$. We shall suppose that the determinant $B$ is not equal to zero.

Multiplying equations $3^{8}$ by $\mathrm{B}_{i 2}, \mathrm{~B}_{i_{3}}, \ldots, \mathrm{B}_{i n}$ and adding we obtain

$$
\mathrm{M}_{i} \mathrm{~B}=\sum_{j=2}^{n} \mathfrak{N}_{j} \mathrm{~B}_{i j}-\mathrm{M}_{1} \sum_{j=2}^{n} m_{x_{j}} \mathrm{~B}_{i j}, \quad i=2,3, \ldots, n .
$$

If we give to $M_{x}$ any value whatever, these equations determine values of $M_{2}, M_{3}, \ldots, M_{n}$, and the values of $M_{x}, M_{2}$, $\ldots, M_{n}$ thus determined, will satisfy equations 33 . To show this, let us multiply equations 39 by $m_{2 k}, m_{3 k}, \ldots, m_{n k}, k$ being one of the numbers $\mathrm{I}, 2, \ldots, n$ and add. We obtain

$$
\mathrm{B} \sum_{i=2}^{n} m_{i k} \mathrm{M}_{i}=\sum_{j=2}^{n} \mathfrak{g} \pi_{j} \sum_{i=2}^{n} m_{i k} \mathrm{~B}_{i j}-\mathrm{M}_{1} \sum_{j=2}^{n} \sum_{\substack{i=2 \\ k=\mathrm{I}, 2, \ldots, n,}}^{n} m_{i k} m_{\mathrm{rj}} \mathrm{B}_{i j},
$$


or, by adding $\mathrm{B} m_{: k} \mathrm{M}_{\mathrm{x}}$ to each side of the equation,

$$
\mathrm{B} \sum_{i=1}^{n} m_{i k} \mathrm{M}_{i}=\sum_{j=2}^{n} \mathfrak{N}_{j} \sum_{i=2}^{n} m_{i k} \mathrm{~B}_{i j}+\mathrm{M}_{1}\left(\mathrm{~B} m_{\mathrm{x} i k}-\sum_{\substack{j=2 \\ k=1}}^{n} \sum_{i=2}^{n} m_{i k} m_{\mathrm{v}} \mathrm{B}_{i j}\right),
$$

The coefficient of $M_{I}$ in the second member of this equation can be written in the form

$$
\begin{array}{ccccc}
m_{1 k}, & m_{2 k}, & m_{3 k}, & \ldots, & m_{n k} \\
m_{12}, & m_{22}, & m_{32}, & \ldots, & m_{n 2} \\
\vdots & \vdots & \vdots & & \vdots \\
m_{1 n}, & m_{2 n}, & m_{3 n}, & \ldots, & m_{n n}
\end{array}
$$

If $k=\mathrm{I}$, this determinant reduces to the determinant $\mathrm{A}$ which, by hypothesis, is equal to zero; and if $k=2,3, \ldots, n$, the elements of two lines become identical, and the determinant again reduces to zero. Thus, for all values of $k$, the coefficient of $\mathrm{M}_{r}$ is equal to zero.

Further, if $k=2,3, \ldots, n$, the coefficient of $\mathfrak{N}_{j}$ :

$$
\sum_{i=2}^{n} m_{i k} \mathrm{~B}_{i j}
$$

is equal to zero unless $j$ is equal to $k$, in which case this coeffcient is equal to $B$. Accordingly we have

$$
\mathrm{B} \sum_{i=1}^{n} m_{i k} \mathrm{M}_{i}=\mathrm{B} \mathfrak{N}_{k}, \quad k=2,3, \cdots n,
$$

If $k=\mathrm{I}$, we shall write the second member of equation 40 in the form

$$
\mathrm{B} \mathfrak{N}_{1}-\left(\mathfrak{N}_{1} \mathrm{~B}-\sum_{j=2}^{n} \sum_{i=2}^{n} \mathfrak{N}_{j} m_{i \mathrm{i}} \mathrm{B}_{i j}\right) .
$$

The quantity within the parenthesis can be written in the determinant form 


$\begin{array}{ccccc}\mathfrak{M T}_{1}, & m_{21}, & m_{31}, & \ldots, & m_{n 1} \\ \mathfrak{M K}_{2}, & m_{22}, & m_{32}, & \ldots, & m_{n 2} \\ \mathfrak{M r}_{3}, & m_{23}, & m_{33}, & \ldots, & m_{n 3} \\ \vdots & \vdots & \vdots & & \vdots \\ \mathfrak{N}_{n}, & m_{2 n}, & m_{3 n}, & \ldots, & m_{n n}\end{array}$

and this is equal to zero in virtue of the first of equations 37 . We have accordingly

$$
\mathrm{B} \sum_{i=\mathrm{I}}^{n} m_{i \mathrm{I}} \mathrm{M}_{i}=\mathrm{B} \mathfrak{N}_{1} .
$$

As we have supposed that $B$ is not equal to zero, we may divide equations $4 \mathrm{I}$ and 42 by that quantity and we obtain equations 33. Thus the values of $\mathrm{M}_{3}, \mathrm{M}_{2}, \ldots, \mathrm{M}_{n}$ which are given by equations 39 do satisfy equations 33 .

When the conditions 36 and 37 are satisfied, the system behaves like a univariant system and we have an indifferent point of the bivariant system. To show this, we recall that, for a bivariant system, two of the quantities $T, \Pi, m_{i j}$ can be chosen arbitrarily; but as equation 36 furnishes a new relation between these quantities, there is now only one which is arbitrary. Moreover, the masses $\mathscr{N}_{j}$ having been chosen so as to satisfy equations 37 , we can choose the mass $M_{x}$ of one of the phases arbitrarily and equations 39 determine the masses of the remaining phases. Thus, at a given temperature and under the corresponding pressure, the equilibrium of the bivariant system is indifferent.

\section{2. Indifferent Points of Trivariant Systems}

Let us consider next a trivariant system, that is, a system for which $r=n-\mathrm{I}$, and let us write equations 30 for this case :

$$
\sum_{i=1}^{n-1} m_{i j} \mathrm{M}_{i}=\mathfrak{N}_{j}, \quad j=\mathrm{I}, 2, \cdots, n .
$$


For the moment, disregard the first of these equations and consider the $n$ - I others :

$$
\sum_{i=1}^{n-I} m_{i j} \mathrm{M}_{i}==\mathfrak{N}_{j}, \quad j=2,3, \ldots, n .
$$

Let us write

$$
\left|\begin{array}{cccc}
m_{12}, & m_{22}, & \ldots, & m_{n-\mathrm{I}, z} \\
m_{13}, & m_{23}, & \cdots, & m_{n-\mathrm{I}, 3} \\
\vdots & \vdots & & \vdots \\
m_{1 n}, & m_{2 n}, & \ldots, & m_{n-\mathrm{I}, n}
\end{array}\right|=\mathrm{C},
$$

and let us denote by $\mathrm{C}_{i j}$ the minor which corresponds to the element $m_{i j}$. Multiplying equations 44 by $\mathrm{C}_{i 2}, \mathrm{C}_{i_{3}}, \ldots, \mathrm{C}_{i n}$ and adding, we find

$$
\mathrm{M}_{i} \mathrm{C}=\sum_{j=2}^{n} \mathfrak{N}, \mathrm{C}_{i j}, \quad i=\mathrm{I}, 2, \ldots, n-\mathrm{I}
$$

Suppose that

$$
\mathrm{C}=\mathrm{o} \text {. }
$$

Then if equations 46 are to be satisfied we must also have

$$
\sum_{j=2}^{n} \mathfrak{M}_{j} \mathrm{C}_{i j}=-=0, \quad i=\mathrm{I}, 2, \ldots, n-\mathrm{I} .
$$

These $n-$ I equations are compatible, for we have

$$
\left|\begin{array}{cccc}
\mathrm{C}_{12}, & \mathrm{C}_{18}, & \cdots, & \mathrm{C}_{1 n} \\
\mathrm{C}_{22}, & \mathrm{C}_{23}, & \cdots, & \mathrm{C}_{2 n} \\
\vdots & \vdots & & \vdots \\
\mathrm{C}_{n-1,2}, & \mathrm{C}_{n-1,3}, & \cdots, & \mathrm{C}_{n-1, n}
\end{array}\right|=\mathrm{C}^{n-2}=0
$$

Accordingly let us suppose that equation 47 is satisfied and that the masses $\mathfrak{N}_{2}, \mathfrak{N}_{3}, \ldots, \mathfrak{N}_{n}$ have been chosen so as to satisfy equations 48 . This can be done in an infinite number of ways since equations 48 determine only the ratios of the masses 
$\mathfrak{N}_{2}, 9 \pi_{3}, \ldots, 9 \mathfrak{T}_{n}$. Let us disregard the first of equations 44 and let us write the others in the form:

$$
\sum_{i=2}^{n-\mathrm{I}} m_{i j} \mathrm{M}_{i}=\mathfrak{N}_{j}-m_{\mathrm{I} j} \mathrm{M}_{1}, \quad j=3,4, \cdots, n .
$$

Let us write

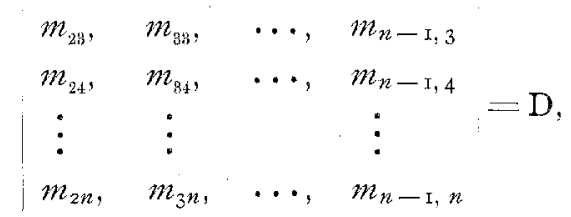

and let us denote by $D_{i j}$ the minor which corresponds to $m_{i j}$. We shall suppose that the determinant $D$ is not equal to zero. Then multiplying equations 49 by $\mathrm{D}_{i_{3}}, \mathrm{D}_{i_{4}}, \ldots, \mathrm{D}_{i n}$ and adding, we get

$$
\mathrm{M}_{i} \mathrm{D}=\sum_{j=3}^{n} \mathscr{M}_{j} \mathrm{D}_{i j}-\mathrm{M}_{1} \sum_{j=3}^{n} m_{1 j} \mathrm{D}_{i j}, \quad i=2,3, \ldots, n-\mathrm{I} . \quad(50)
$$

If we give to $\mathrm{M}_{\mathrm{r}}$ any value whatever, these equations will determine the corresponding values of $\mathrm{M}_{2}, \mathrm{M}_{3}, \ldots, \mathrm{M}_{n-\mathrm{r}}$.

Let us see now under what conditions these values of $M_{r}$, $\mathrm{M}_{2}, \ldots, \mathrm{M}_{n-1}$ will satisfy equations 43 . Multiply equations 50 by $m_{2 k}, m_{3 k}, m_{n-\mathrm{I}, k}, k$ being one of the numbers I, $2, \ldots, n$, and add. We get

$$
\mathrm{D} \sum_{i=2}^{n-1} m_{i k} \mathrm{M}_{i}=\sum_{j=3}^{n} \mathfrak{N}_{j} \sum_{i=2}^{n-1} m_{i k} \mathrm{D}_{i j}-\mathrm{M}_{1} \sum_{j=3}^{n} \sum_{i=2}^{n-1} m_{1 j} m_{i k} \mathrm{D}_{i j}, \underbrace{}_{k=\mathrm{I}, 2, \ldots, n,}
$$

or, adding $\mathrm{D} m_{I k} \mathrm{M}_{I}$ to each side of the equation,

$$
\begin{aligned}
& \mathrm{D} \sum_{i=1}^{n-\mathrm{I}} m_{i k} \mathrm{M}_{i}=\sum_{j=3}^{n} \mathfrak{N}_{j} \sum_{i=2}^{n-\mathrm{I}} m_{i k} \mathrm{D}_{i j}+\mathrm{M}_{1}\left(m_{\mathrm{L} k} \mathrm{D}-\sum_{j=3}^{n} \sum_{i=2}^{n-\mathrm{I}} m_{\mathrm{J}} m_{i k} \mathrm{D}_{i j}\right), \\
& k=\mathrm{I}, 2, \ldots, n . \quad \text { (5. I }
\end{aligned}
$$


The coefficient of $\mathrm{M}_{\mathrm{T}}$ in the second member of this equation can be put into the determinant form

$$
\left|\begin{array}{ccccc}
m_{1 k}, & m_{2 k}, & m_{3 k}, & \ldots, & m_{n-1, k} \\
m_{13}, & m_{23}, & m_{33}, & \ldots, & m_{n-1,3} \\
m_{14}, & m_{24}, & m_{3+}, & \ldots, & m_{n-1,4} \\
\vdots & \vdots & \vdots & & \vdots \\
m_{1 n}, & m_{2 n}, & m_{3 n}, & \ldots, & m_{n-1, n}
\end{array}\right|
$$

Now, if $k=3,4, \ldots, n$, the elements of two lines of this determinant become identical and the determinant reduces to zero. If $k=2$, this determinant is the determinant $\mathrm{C}$ which, by hypothesis, is equal to zero. If $k=\mathrm{I}$, we shall have a determinant different from $\mathrm{C}$, but which we shall also suppose to be equal to zero. We thus introduce the new condition

$$
\mid \begin{array}{cccc}
m_{11}, & m_{21}, & \ldots, & m_{n-1, I} \\
m_{13}, & m_{23}, & \ldots, & m_{n-1,3} \\
\vdots & \vdots & & \vdots \\
m_{1 n}, & m_{2 n}, & \ldots, & m_{n-1, n}
\end{array}==0 .
$$

Thus for all the values of $k$, the term in $M_{T}$ disappears from the second member of equation $5 \mathrm{I}$.

The coefficient of $\mathfrak{N}_{j}$ :

$$
\sum_{i=2}^{n-1} m_{i k} \mathrm{D}_{i j}
$$

will be equal to zero for $k=3,4, \ldots, n$, except when $j$ is equal to $k$, in which case it is equal to $D$. We have thus

$$
\mathrm{D} \sum_{i=1}^{n-1} m_{i k} \mathrm{M}_{i}=\mathrm{D}^{\mathrm{N}} \boldsymbol{n}_{k}, \quad k=3,4, \cdots, n
$$

When $k=2$, we shall write the second member of equation 5 in the form 


$$
\mathrm{D} \mathfrak{N}_{2}-\left(\mathfrak{N}_{2} \mathrm{D}-\sum_{j=3}^{n} \sum_{i=2}^{n-x} \mathfrak{N}_{j} m_{i_{2}} \mathrm{D}_{i j}\right) .
$$

The quantity within the parenthesis can be written in the determinant form

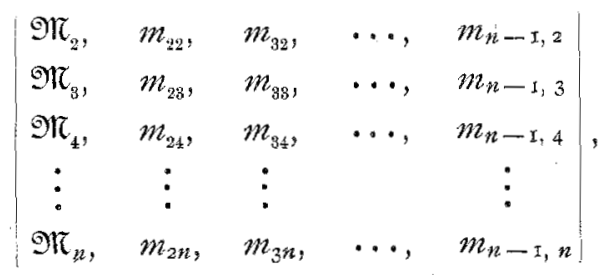

and the first of equations 48 shows that this determinant is equal to zero. We have thus

$$
\mathrm{D} \sum_{i=1}^{n-\mathrm{I}} m_{i_{2}} \mathrm{M}_{i}=\mathrm{D} \mathfrak{M}_{2}
$$

Similarly, when $k=\mathrm{I}$, we can write the second nember of equation $5 \mathrm{I}$ in the form

$$
\mathrm{D} \mathscr{N} \mathbb{K}_{1}-\left|\begin{array}{ccccc}
\mathscr{N}_{1}, & m_{21}, & m_{31}, & \ldots, & m_{n-\mathrm{I}, \mathrm{I}} \\
\mathfrak{N}_{3}, & m_{23}, & m_{33}, & \ldots, & m_{n-\mathrm{I}, 3} \\
\vdots & \vdots & \vdots & & \vdots \\
\mathfrak{N}_{n}, & m_{2 n}, & m_{3 n}, & \cdots, & m_{n-\mathrm{I}, n}
\end{array}\right|
$$

If then, we choose the mass $\mathfrak{N K}_{\mathrm{I}}$ so that

$$
\left[\begin{array}{ccccc}
\mathfrak{M}_{1}, & m_{21}, & m_{31}, & \cdots, & m_{n-\mathrm{I}, \mathrm{I}} \\
\mathfrak{N K}_{3}, & m_{23}, & m_{33}, & \ldots, & m_{n-\mathrm{I}, 3} \\
\vdots & \vdots & \vdots & & \vdots \\
\mathfrak{M H}_{n}, & m_{2 n}, & m_{3 n}, & \ldots, & m_{n-\mathrm{I}, n}
\end{array}\right.
$$

we shall have

$$
\mathrm{D} \sum_{i=\mathrm{I}}^{n-\mathrm{I}} m_{i \mathrm{I}} \mathrm{M}_{i}=\mathrm{D} 9 \pi_{\mathrm{L}}
$$


As. $D$ is not equal to zero, we may divide equations 53,54 and 56 by that quantity; we thus obtain equations 43 . Thus the values of $M_{1}, M_{2}, \ldots, M_{n-1}$, which are given by equations 50 , do satisfy equations 43 .

The trivariant system now behaves like a univariant system. To show this, we observe that for a trivariant system three of the variables $T, \Pi, m_{i j}$ are independent. But we have just imposed upon those variables the two new conditions 47 and 52 ; there is thus but one which is independent. Further, the masses of the components having been chosen so as to satisfy equations 48 and 55 , we can choose the mass $M_{z}$ arbitrarily and the masses of the other phases will be determined by equations 5o. Thus, whenever the conditions $47,52,48$ and 55 are satisfied we shall have an indifferent point of the trivariant system.

Equations $47,52,48$ and 55 have as consequences certain other equations which will enable us to write the conditions for an indifferent point in a symmetric form. To show this, let us denote the determinant that appears in equation 52 , by $\mathrm{C}^{\prime}$, and by $\mathrm{C}_{i j}^{\prime}$, the minor which corresponds to the element $m_{i j}$, and let us consider the equations which we obtain by omitting the second of equations 43 :

$$
\sum_{i=1}^{n-\mathrm{I}} m_{i j} \mathrm{M}_{i}=\mathfrak{M N}_{j}, \quad j=\mathrm{I}, 3, \cdots, n
$$

Multiplying these equations by $\mathrm{C}^{\prime}{ }_{i 1}, \mathrm{C}^{\prime}{ }_{i_{3}}, \ldots, \mathrm{C}^{\prime}{ }_{\text {in }}$ and adding, we find

$$
\mathrm{M}_{i} \mathrm{C}^{\prime}=\sum_{j=\mathrm{r}, 3}^{n} 9 \mathfrak{n}_{j} \mathrm{C}_{i j}^{\prime}=\mathrm{O}, \quad i=\mathrm{I}, 2, \cdots, n-\mathrm{I} .
$$

As $\mathrm{C}^{\prime}$ is equal to zero, it follows that we must have also

$$
\sum_{j=\mathrm{I}, 3}^{n} \mathfrak{M}_{j} \mathrm{C}_{i j}^{\prime}=0, \quad i=\mathrm{I}, 2, \ldots, n-\mathbf{I} .
$$

The first of these equations is equation 55 . 
In like manner let us consider the equations which we obtain by omitting the third of equations 43 :

$$
\sum_{i=\mathrm{I}}^{n-\mathrm{I}} m_{i j} \mathrm{M}_{i}=\mathfrak{N}_{j}, \quad j=\mathrm{I}, 2,4, \cdots, n .
$$

Let us write

$$
\begin{array}{cccc}
m_{11}, & m_{21}, & \ldots, & m_{n-\mathrm{I}, \mathrm{I}} \\
m_{12}, & m_{22}, & \ldots, & m_{n-\mathrm{I}, 2} \\
m_{14}, & m_{24}, & \ldots, & m_{n-\mathrm{I}, 4} \\
\vdots & \vdots & & \vdots \\
m_{1 n}, & m_{2 n}, & \ldots, & m_{n-\tau, n}
\end{array}
$$

and let us denote by $\mathrm{C}^{\prime \prime}{ }_{i j}$ the minor which corresponds to the element $m_{i j}$. Multiplying equations 60 by $\mathrm{C}^{\prime \prime}{ }_{i 1}, \mathrm{C}^{\prime \prime}{ }_{i 2}, \mathrm{C}^{\prime \prime}{ }_{i 4}, \ldots$, $\mathrm{C}^{\prime \prime}{ }_{\text {in }}$ and adding, we obtain

$$
\mathrm{M}_{i} \mathrm{C}^{\prime \prime}=\sum_{j=1,2,4}^{n} \mathfrak{N}_{j} \mathrm{C}^{\prime \prime}{ }_{i j}, \quad i=\mathrm{I}, 2, \ldots, n-\mathrm{I}
$$

As $\mathrm{M}_{i}$ admits of an infinite number of values it follows that we must have

and

$$
\mathrm{C}^{\prime \prime}=\mathrm{o}
$$

$$
\sum_{j=\mathrm{I}, 2,4}^{n} \mathfrak{M}_{j} \mathrm{C}_{i j}^{\prime \prime}=0, \quad i=\mathrm{I}, 2, \ldots, n-\mathrm{I} .
$$

By continuing this reasoning, we see that whenever the trivariant system is in a state of indifferent equilibrium, all the determinants of order $n$ - I which can be formed from the following matrix :

$$
\begin{array}{ccccc}
\mathfrak{N K}_{1}, & m_{11}, & m_{21}, & \ldots, & m_{n-\mathrm{I}, \mathrm{I}} \\
\mathfrak{N K}_{2}, & m_{12}, & m_{22}, & \ldots, & m_{n-\mathrm{I}, 2} \\
\vdots & \vdots & \vdots & & \vdots \\
\mathfrak{N K}_{n}, & m_{1 n}, & m_{2 n}, & \ldots, & m_{n-\mathrm{I}, n}
\end{array}
$$

are equal to zero. 
8. Indifferent Points of Systems of Variance $p+2$.

Let us consider now a multivariant system of variance, $p+2$, that is, a system for which $r=n-p$. By reasoning similar to that used in discussing trivariant systems, we can show that we shall have an indifferent point of the system whenever the concentrations satisfy the $p+$ I equations:

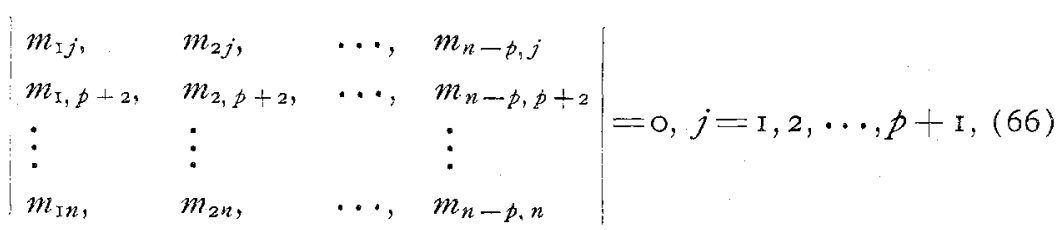

and the masses of the components satisfy the equations :

$$
\begin{aligned}
& \left|\begin{array}{cccc}
\mathfrak{N}_{p+\mathrm{I}}, & m_{2, p+\mathrm{I}}, & \cdots, & m_{n-p, p+\mathrm{I}} \\
\mathfrak{N}_{p+2}, & m_{2, p+2}, & \cdots, & m_{n-p, p+2} \\
\vdots & \vdots & & \vdots \\
\mathfrak{N}_{n}, & m_{2 n}, & \cdots, & m_{n-p, n}
\end{array}\right|=0, \\
& \left|\begin{array}{cccc}
m_{\mathrm{r}, p+\mathrm{I}}, & \mathfrak{N K}_{p \div \mathrm{I}}, & \cdots, & m_{n-p, p+\mathrm{I}} \\
m_{\mathrm{r}, p+2}, & \mathfrak{N R}_{p+2}, & \cdots, & m_{n-p, p+2} \\
\vdots & \vdots & & \vdots \\
m_{1 n}, & \operatorname{NR}_{n}, & \cdots, & m_{n-p, n}
\end{array}\right|=0 \\
& \left|\begin{array}{cccc}
m_{\mathrm{I}, p+\mathrm{I}}, & m_{2, p+\mathrm{I}}, & \cdots, & \mathfrak{M}_{p+\mathrm{I}} \\
m_{\mathrm{I}, p+2}, & m_{2, p+2}, & \cdots, & \mathfrak{M}_{p+2} \\
\vdots & \vdots & & \vdots \\
m_{\mathrm{I} n}, & m_{2 n}, & \cdots, & \mathfrak{M}_{n}
\end{array}\right|=0 \\
& \begin{array}{llll}
\mathscr{N}_{j,} & m_{2 j}, & \cdots, & m_{n-p, j} \\
\mathscr{N}_{p+2} & m_{2, p+2} & \cdots, & m_{n-p, p+2} \\
\vdots & \vdots & & \vdots \\
\mathfrak{N}_{n}, & m_{2 n} & \cdots, & m_{n-p, n}
\end{array}
\end{aligned}
$$


When $p=0$, these conditions reduce to conditions 36 and 37, which we have found for bivariant systems, and when $p=1$, they reduce to the conditions $47,52,48$ and 55 of trivariant systems.

Further, by reasoning similar to that used for trivariant systems, we can show that, whenever the multivariant system is in a state of indifferent equilibrium, all the determinants of order $n-p$ that can be formed from the matrix

$$
\begin{array}{ccccc}
\mathfrak{M K}_{1}, & m_{11}, & m_{21}, & \ldots, & m_{n-p, 1} \\
\mathfrak{N K}_{2}, & m_{12}, & m_{22}, & \ldots, & m_{n-p, 2} \\
\vdots & \vdots & \vdots & & \vdots \\
\mathfrak{N K}_{n}, & m_{1 n}, & m_{2 n}, & \ldots, & m_{n-p, 2 v}
\end{array}
$$

are equal to zero.

If the temperature or the pressure of a bivariant or multivariant system be given, and if the variables $T, \Pi, m_{i j}$ satisfy conditions 66 and the masses of the components satisfy conditions 67 , the equilibrium of the bivariant or multivariant system will be indifferent and the mass of one of the phases can be chosen arbitrarily. If the volume of the system be also given, equation 32 shows that the masses of all the phases will be determined; the equilibrium will no longer be indifferent. Thus the state of equilibrium of a bivariant or multivariant system at an indifferent point will be completely determined if the temperature and the volume or the pressure and the volume are known.

It follows from this discussion and from that given at the end of the last chapter that, with the exception of invariant systems, the state of equilibrium of every other system is determined when the temperature and the volume or the pressure and the volume are known.

There is a property of indifferent points which should be mentioned here. Let us consider a multivariant system of $r$ phases formed by means of $n$ independent components. If the system is at an indifferent point, we know that all the determinants of order $r$ that can be formed from the matrix 


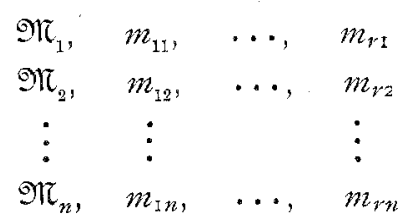

are equal to zero. Consider now a second system which differs from the system of $r$ phases, in indifferent equilibrium, by the addition of a new phase formed from the same components. The equilibrium of the new system, if it be possible, will be indifferent. For this to be so, we know that all the determinants of order $r+$ I which can be formed from the matrix

$$
\begin{array}{ccccc}
\mathfrak{N}_{1}, & m_{11}, & \ldots, & m_{r 1}, & m_{r-\mathrm{I}, \mathrm{I}} \\
\mathfrak{N K}_{2}, & m_{12}, & \ldots, & m_{r_{2},} & m_{r+\mathrm{I}, 2} \\
\vdots & \vdots & & \vdots & \vdots \\
\mathfrak{N K}_{n,} & m_{1 n}, & \ldots, & m_{r n}, & m_{r+1, n}
\end{array}
$$

must be equal to zero. But it is easy to see that all these determinants reduce to zero, for each determinant of order $r+\mathrm{I}$ can be developed into a sum of determinants of order $r$, each of which is, by hypothesis, equal to zero. Thus the indifferent points of a multivariant system of $r$ phases are also indifferent points of a bivariant or multivariant system of $r-$ I phases.

\section{Clapeyron's Equation}

The total thermodynamic potential of the $i$-th phase is given by the equation

$$
\Phi_{i}=\sum_{j=\mathrm{I}}^{n} \mathrm{~F}_{i j} \mathrm{M}_{i j},
$$

and we know that, when the system is in equilibrium,

$$
\frac{\partial \Phi_{i}}{\partial \mathrm{T}}=-\mathrm{H}_{i}, \quad \frac{\partial \Phi_{i}}{\partial I}=V_{i} .
$$

Consider an infinitesimal reversible change of the $i$-th phase, that is, consider two adjacent states of equilibrium. The change 
which the potential of the phase undergoes can be written in the following ways:

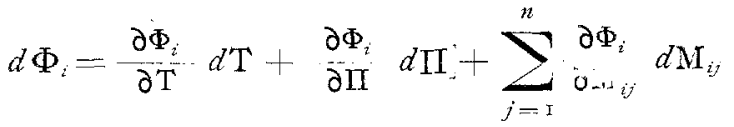

$$
\begin{aligned}
& =-\mathrm{H}_{i} d \mathrm{~T}^{\prime}+\mathrm{V}_{i} d \Pi+\sum_{j=\mathrm{I}}^{n} \mathrm{~F}_{i j} d \mathrm{M}_{i j}, \\
& d \Phi_{i}=\sum_{j=\mathrm{I}}^{n} \mathrm{~F}_{i j} d \mathrm{M}_{i j}+\sum_{j=\mathrm{I}}^{n} \mathrm{M}_{i j} d \mathrm{~F}_{i j} .
\end{aligned}
$$

The comparison of equations 69 and 70 yields at once the equation

$$
\mathrm{V}_{i} d \Pi=\mathrm{H}_{i} d \mathrm{~T}+\sum_{j=1}^{n} \mathrm{M}_{i j} d \mathrm{~F}_{i j} .
$$

Let us divide this equation by the mass $M_{i}$ and let us denote by $v_{i}$ and $\eta_{i}$ the volume and the entropy of the unit of mass of the $i$-th phase; we thus obtain

$$
v_{i} d \Pi=\eta_{i} d \mathrm{~T}+\sum_{j=\mathrm{I}}^{n} m_{i j} d \mathrm{~F}_{i j} .
$$

When the system of $r$ phases is in equilibrium, conditions 25 are satisfied. We may write then in the form

$$
\mathrm{F}_{r j}=\mathrm{F}_{2 j}=\cdots=\mathrm{F}_{r j}=\mathrm{F}_{j}, \quad j=\mathrm{I}, 2, \ldots, n,
$$

in which $\mathrm{F}_{j}$ denotes the common value of the functions $\mathrm{F}_{i j}$ which correspond to the $j$-th component. In a reversible change, equations 72 are constantly satisfied; accordingly, for an infinitesimal reversible change we shall have

$$
d \mathrm{~F}_{1 j}=d \mathrm{~F}_{z j}=\ldots=d \mathrm{~F}_{r j}=d \mathrm{~F}_{j}, \quad j=\mathrm{I}, 2, \ldots, n,
$$

and we can write equations $7 \mathrm{I}$ in the form

$$
v_{i} d \Pi==\eta_{i} d \mathrm{~T}+\sum_{j=1}^{n} m_{i j} d \mathrm{~F}_{j}, \quad i=\mathrm{I}, 2, \ldots, r .
$$


If the system under consideration is invariant, $d \mathrm{~T}, d \mathrm{II}$ and $d \mathrm{~F}_{j}$ are equal to zero and all the terms of equation 74 vanish. In every other case, however, we can eliminate, from the $r$ equations $74, r-\mathrm{I}$ of the quantities $d \Pi, d \mathrm{~T}, d \mathrm{~F}_{j}$. If the system is univariant, $r$ is equal to $n+\mathrm{I}$, and we can eliminate the $n$ quantities $d \mathrm{~F}_{j}$, We thus obtain

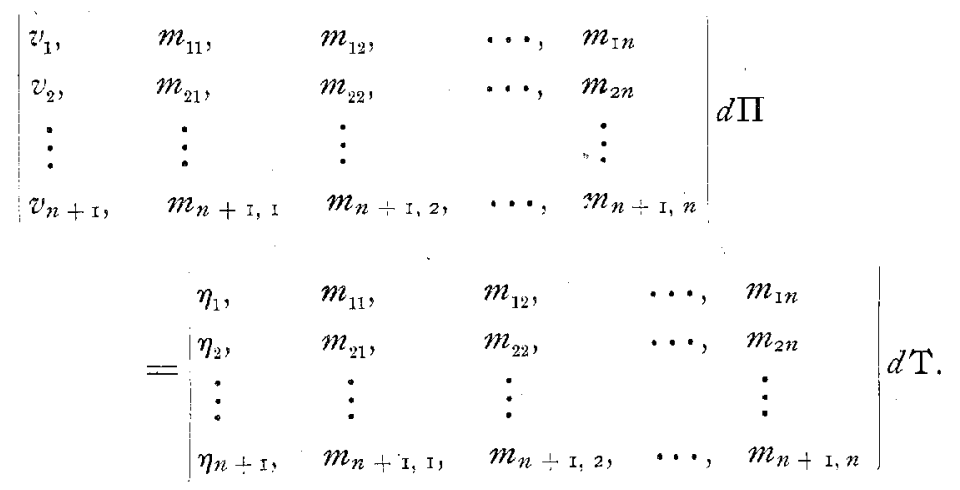

If the system is bivariant, $r$ is equal to $n$, and we can eliminate $n-\mathrm{I}$ of the quantities $d \mathrm{~F}_{j}$, for example $d \mathrm{~F}_{2}, d \mathrm{~F}_{3}$, $\ldots, d \mathrm{~F}_{n}$. We thus obtain

$$
\begin{aligned}
& \left|\begin{array}{ccccc}
v_{1}, & m_{12}, & m_{13}, & \ldots, & m_{1 n} \\
v_{2}, & m_{212}, & m_{23}, & \ldots, & m_{2 n} \\
\vdots & \vdots & \vdots & & \vdots \\
v_{n}, & m_{n 2}, & m_{n 3}, & \ldots, & m_{n n}
\end{array}\right| d \Pi=\left|\begin{array}{cccll}
\eta_{1}, & m_{12}, & m_{13}, & \ldots, & m_{1 n} \\
\eta_{2}, & m_{22}, & m_{23}, & \ldots, & m_{2 n} \\
\vdots & \vdots & \vdots & & \vdots \\
\eta_{n}, & m_{n 2}, & m_{n 3}, & \ldots, & m_{n n}
\end{array}\right| d \mathrm{~T} \\
& \begin{array}{cccc}
m_{11}, & m_{12}, & \cdots, & m_{1 n} \\
+m_{21}, & m_{22}, & \cdots, & m_{2 n} \\
\vdots & \vdots & & \vdots \\
m_{n 1}, & m_{n 2}, & \cdots, & m_{n n}
\end{array} \mid d \mathrm{~F}_{1}
\end{aligned}
$$

Finally, for a multivariant system of variance $p+2, r$ is equal to $n-p$. Eliminating the $n-p-\mathrm{I}$ quantities $d \mathrm{~F}_{p+2}$, $d \mathrm{~F}_{p-3}, \ldots, d \mathrm{~F}_{n}$, we find 


$$
\begin{aligned}
& \left|\begin{array}{cccc}
v_{1}, & m_{1, p+2,}, & \cdots, & m_{\mathrm{I} n} \\
v_{2}, & m_{2, p+2,} & \cdots, & m_{2 n} \\
\vdots & \vdots & & \vdots \\
v_{n-p}, & m_{n-p, p \div 2}, & \cdots, & m_{n-p, n}
\end{array}\right| d \Pi \\
& =\left|\begin{array}{cccc}
\eta_{1}, & m_{\mathrm{I}, p+2}, & \ldots, & m_{1 n} \\
\eta_{2}, & m_{2, p+2}, & \ldots, & m_{2 n} \\
\vdots & \vdots & & \vdots \\
\eta_{n-p}, & m_{n-p, p \div 2}, & \ldots, & m_{n-p, n}
\end{array}\right| d \mathrm{~T} \\
& +\sum_{j=1}^{p+1}\left|\begin{array}{cccc}
m_{1 j}, & m_{1, p+2}, & \ldots, & m_{1 n} \\
m_{2 j}, & m_{2, p+2}, & \ldots, & m_{2 n} \\
\vdots & \vdots & & \vdots \\
m_{n-p, j}, & m_{n-p, p+2}, & \cdots, & m_{n-p, n}
\end{array}\right| d \mathrm{~F}_{j} .
\end{aligned}
$$

When $p=0$, equation 77 reduces to equation 76 .

Gibbs has shown, ${ }^{2}$ in the particular case of a univariant system formed from one component, that equation 75 can be put into the form of Clapeyron's equation, and that at an indifferent point of a bivariant system, formed by means of two components, equation 76 can be written in the same form. We shall now show that, for any univariant system, it is possible to write equation 75 in the form of Clapeyron's equation, and that for any bivariant or multivariant system at an indifferent point, equation 77 can be written in the same form.

Consider first a univariant system. At a given temperature and under the corresponding pressure, the system admits of a continuous series of states of equilibrium in which the masses of the phases vary while the concentrations remain unchanged. Let us denote by $\mathrm{M}_{i}^{\prime}$ and $\mathrm{M}_{i}$ the mass of the $i$-th phase, in two of these states of equilibrium, and by $\mathrm{V}^{\prime}$ and $\mathrm{V}, \mathrm{H}^{\prime}$ and $\mathrm{H}$, the volume and the entropy of the system in these two states. If the masses of the components remain unchanged, equations 30 show that we may write

' On the Equilibrium of Heterogeneous Substances, p. I55. 


$$
\sum_{i=1}^{n+\mathrm{i}} m_{i j}\left(\mathrm{M}_{i}^{\prime}-\mathrm{M}_{i}\right)=0, \quad j=\mathrm{I}, 2, \ldots, n .
$$

In like manner the consideration of equation 32 and of the analogous equation

$$
\mathrm{H}=\sum_{i=1}^{n+\mathrm{I}} \mathrm{H}_{i}=\sum_{i=1}^{n+\mathrm{I}} \eta_{i} \mathrm{M}_{2}
$$

shows that we may write

$$
\begin{aligned}
& \sum_{i=1}^{n+\mathrm{I}} v_{i}\left(\mathrm{M}_{i}^{\prime}-\mathrm{M}_{i}\right)=\mathrm{V}^{\prime}-\mathrm{V}, \\
& \sum_{i=1}^{n+\mathrm{I}} \eta_{i}\left(\mathrm{M}_{i}^{\prime}-\mathrm{M}_{i}\right)=\mathrm{H}^{\prime}-\mathrm{H} .
\end{aligned}
$$

If, from the $n+2$ equations 78,80 and 8 I we eliminate the $n+$ I quantities $\mathrm{M}_{i}^{\prime}-\mathrm{M}_{i}$, we obtain

$$
\begin{array}{cccccc}
\mathrm{H}^{\prime}-\mathrm{H}, & \mathrm{V}^{\prime}-\mathrm{V}, & 0, & 0, & \ldots, & 0 \\
\eta_{1}, & v_{1}, & m_{11}, & m_{12}, & \ldots, & m_{1 n} \\
\eta_{2}, & v_{2}, & m_{21}, & m_{22}, & \ldots, & m_{2 n} \\
\vdots & \vdots & \vdots & \vdots & \vdots & \vdots \\
\eta_{n+1}, & \tau_{n+\mathrm{I}}, & m_{n+\mathrm{I}, \mathrm{I}}, & m_{n+\mathrm{I}, 2}, & \ldots, & m_{n-\mathrm{I}, n}
\end{array}
$$

or by developing,

$$
\begin{aligned}
& \left(\mathrm{H}^{\prime}-\mathrm{H}\right) \begin{array}{cccc}
v_{1}, & m_{11}, & \cdots, & m_{1 n} \\
v_{2}, & m_{21}, & \ldots, & m_{2 n} \\
\vdots & \vdots & & \vdots \\
v_{n+1}, & m_{n+\mathrm{I}, \mathrm{I}}, & \ldots, & m_{n+\mathrm{I}, n}
\end{array} \\
& =\left(\mathrm{V}^{\prime}-\mathrm{V}\right)\left|\begin{array}{llll|}
\eta_{1}, & m_{11}, & \ldots, & m_{1 n} \\
\eta_{2}, & m_{21}, & \ldots, & m_{2 n} \\
\vdots & \vdots & & \vdots \\
\eta_{n+1}, & m_{n+\mathrm{I}, 1}, & \ldots, & m_{n-\mathrm{I}, n}
\end{array}\right|
\end{aligned}
$$


If we compare this equation with equation 75 , we get at once

$$
\frac{d \mathbf{I I}}{d^{\prime} \mathrm{T}}=\frac{\mathrm{H}^{\prime}-\mathrm{H}}{\mathrm{V}^{\prime}-\mathrm{V}} .
$$

If we denote by $Q$ the quantity of heat which the system absorbs in passing from the state $H, V$ to the state $\mathrm{H}^{\prime}, \mathrm{V}^{\prime}$, we have

$$
Q=\top\left(H^{\prime}-\mathrm{H}\right) \text {, }
$$

and equation 84 takes the well-known form

$$
\frac{d \mathbf{I}}{d^{\prime} \mathrm{T}}=\frac{\mathrm{Q}}{\mathrm{T}\left(\mathrm{V}^{\prime}-\mathrm{V}\right)} \text {. }
$$

Consider now a bivariant or multivariant system at an indifferent point. At a given temperature and under the pressure which corresponds to the indifferent point, the system admits of a continuous series of states of equilibrium in which the masses of the phases vary while the concentrations remain unchanged. If the variance is equal to $p+2$, we can write the following equations analogons to 78,80 and $8 \mathrm{I}$,

$$
\begin{aligned}
& \sum_{\substack{i=1 \\
n=p}}^{n-p} m_{i j}\left(\mathrm{M}_{i}^{\prime}-\mathrm{M}_{i}\right)=0, \quad j=\mathrm{I}, 2, \ldots, n, \\
& \sum_{i=1}^{n} v_{i}\left(\mathrm{M}_{i}^{\prime}-\mathrm{M}_{i}\right)=\mathrm{V}^{\prime}-\mathrm{V}, \\
& \sum_{i=1}^{n-p} \eta_{i}\left(\mathrm{M}_{i}^{\prime}-\mathrm{M}_{i}\right)=\mathrm{H}^{\prime}-\mathrm{H} .
\end{aligned}
$$

Then, disregarding the $p+\mathrm{I}$ equations 87 which correspond to $j=\mathrm{I}, 2, \ldots, p+\mathrm{I}$, we can eliminate from the $n-p-\mathrm{I}$ remaining equations 87 and equations 88 and 89 , the $n-p$ quantities $\mathrm{M}^{\prime}{ }_{i}-\mathrm{M}_{i}$. We thus obtain

$$
\begin{array}{cccccl}
\mathrm{H}^{\prime}-\mathrm{H}, & \mathrm{V}^{\prime}-\mathrm{V}, & 0, & \mathrm{O}, & \cdots, & 0 \\
\eta_{1}, & v_{1}, & m_{\mathrm{I}, p+2}, & m_{1, p+3}, & \cdots, & m_{1 n} \\
\eta_{2}, & v_{2}, & m_{2, p+2,}, & m_{2, p+3}, & \cdots, & m_{2 n} \\
\vdots & \vdots & \vdots & \vdots & & \vdots \\
\eta_{n-p}, & v_{n-p}, & m_{n-p, p+2}, & m_{n-p, p-3}, & \cdots, & m_{n-p, n}
\end{array}
$$


or, developing,

$$
\begin{aligned}
& \left(\mathrm{H}^{\prime}-\mathrm{H}\right) \mid \begin{array}{llll}
v_{1}, & m_{\mathrm{I}, p+2}, & \cdots, & m_{\mathrm{In}} \\
\vdots & m_{2, p \div 2}, & \cdots, & m_{212} \\
\vdots & \vdots & & \vdots \\
v_{n-p}, & m_{n-p, p-2}, & \cdots, & m_{n-p, n}
\end{array} \\
& =\left(\mathrm{V}^{\prime}-\mathrm{V}\right)\left|\begin{array}{llll}
\eta_{1}, & m_{\mathrm{I}, p+2,} & \ldots, & m_{\mathrm{I} n} \\
\eta_{2}, & m_{2, p+2}, & \ldots, & m_{2 n} \\
\vdots & \vdots & & \vdots \\
\eta_{n-p}, & m_{n-p, p+2}, & \ldots, & m_{n-p, n}
\end{array}\right| .(9 \mathrm{I})
\end{aligned}
$$

Comparing this equation with equation 77 and noticing that at an indifferent point the terms in $d F_{j}$ vanish in virtue of conditions 66 , we see at once that

$$
\frac{d \mathrm{I}}{d \mathrm{~T}}=\frac{\mathrm{H}^{\prime}-\mathrm{H}}{\mathrm{V}^{\prime}-\mathrm{V}} .
$$

If we denote by $Q$ the quantity of heat which the system absorbs during the reversible change from the state $\mathrm{H}, \mathrm{V}$, to the state $\mathrm{H}^{\prime}, \mathrm{V}^{\prime}$, we have

$$
Q=T\left(H^{\prime}-\mathrm{H}\right),
$$

and equation 92 takes the form of Clapeyron's equation :

$$
\frac{d \Pi}{d T}=\frac{Q}{T\left(V^{\prime}-V\right)} .
$$

Equation 94 completes the analogy which exists between a univariant system and a bivariant or multivariant system at an indifferent point. In both cases, the temperature and the pressure are connected by an equation and the derivative of the pressure with respect to the temperature is given by Clapeyron's formula. Moreover, at a given temperature and under the corresponding pressure, each of the systems admits of a continuous series of states of equilibrium in which the masses of the phases vary while the concentrations remain unchanged. 


\section{The Theorems of Gibbs and Konowalow}

Equation 77 furnishes two more important theorems. Consider a reversible isothermal change of a bivariant or multivariant system. $d \mathrm{~T}$ being equal to zern, the first term of the second member of equation 77 disappears from the equation. If the system passes through an indifferent point, the coefficients of the terms in $d \mathrm{~F}$, will reduce to zero in virtue of conditions 66 . If we suppose that the coefficient of $d \Pi$ is not equal to zero, which, by equation $9 \mathrm{I}$, is equivalent to the supposition that $\mathrm{V}^{\prime}-\mathrm{V}$ is not equal to zero, we shall have

$$
d \Pi=\mathrm{o} .
$$

This means, in general, that the pressure passes through a maximum or a minimum of the values which are possible at the given temperature. Thus, at a given temperature, the pressure of a bivariant or multivariant system at the indifferent point which corresponds to that temperature, will be, in general, a maximum or a minimum of the pressures possible at the given temperature.

In like manner, it can be shown that, under a given pressure, the temperature of a bivariant or multivariant system at the indifferent point which corresponds to that pressure, will be, in general, a maximum or a minimum of the temperatures possible under the given pressure. The demonstration requires the coefficient of $d$ T to be different from zero, which, by equation 9I, is equivalent to the supposition that $\mathrm{H}^{\prime}-\mathrm{H}$ is not equal to zero.

Gibbs has established these two theorems in the case of a bivariant system, and we have simply reproduced his demonstration, applying it, however, to the general equation 77 instead of restricting it to equation 76 . Konowalow has rediscovered ${ }^{2}$ the first of Gibbs's theorems in the particular case of a bivariant system formed by means of two independent components.

I On the Equilibrium of Heterogeneous Substances, p. $\mathrm{r}_{55}$.

${ }^{2}$ Wied. Ann. I4, 48 (I88I). 
CHAPTER III.

\section{THE DISPLACEMENT OF EQUILIBRIUM}

In the last chapter we have established several theorems relating to the displacement of equilibrium, namely, the theorems of Clapeyron and of Gibbs. We shall now treat the question of the displacement of equilibrium in a more general manner and shall show how, from one and the same formula can be deduced the principal theorems relating to the displacement of equilibrium, namely, the theorems of Clapeyron, of Gibbs, of Le Châtelier and of van ' $t$ Hoff.

Let us consider a system of $r$ phases formed by means of $n$ independent components. The total thermodynamic potential of the $i$-th phase satisfies the equation:

$$
\Phi_{i}=\sum_{j=\mathrm{r}}^{n} \frac{\partial \Phi^{i}}{\partial \mathrm{M}_{i j}} \dot{\mathrm{M}}_{i j} .
$$

If we adopt a notation slightly different from that employed in the preceding chapters and write

$$
F_{i j}=\frac{\partial \Phi_{i}}{\partial V_{i j}},
$$

we shall have for the total potential of the $i$-th phase:

$$
\Phi_{i}=\sum_{j=1}^{n} \mathrm{~F}_{i j} \mathrm{M}_{i j},
$$

and for the total potential of the system

$$
\Phi=\sum_{i=\mathrm{I}}^{r} \sum_{j=\mathrm{I}}^{n} \mathrm{~F}_{i j} \mathrm{M}_{i j}
$$

The system will be in stable equilibrium, if, for every virtual change which leaves the temperature, the pressure and the mass of the system unchanged, the total potential of the system increases. If for certain virtual changes the total potential remains constant while for all the others it increases the system 
will be in a state of indifferent equilibrium. Let us impose upon the system a virtual change which leaves the temperature, the pressure and the mass of the system unchanged; if we neglect terms of orders higher than the second, the total potential of the system will become:

$$
\Phi+\sum_{i=1}^{r} \sum_{j=1}^{n} \mathrm{~F}_{i j} \delta \mathrm{M}_{i j}+\frac{1}{2} \sum_{i=1}^{r} \sum_{j=1}^{n} \sum_{k=1}^{n} \frac{\partial \mathrm{F}_{i}}{\partial \mathrm{M}_{i k}} \delta \mathrm{M}_{i j} \delta \mathrm{M}_{i z} .
$$

The system will accordingly be in stable equilibrium if we have:

$$
\sum_{i=1}^{r} \sum_{j=1}^{n} \mathrm{~F}_{i j} \delta \mathrm{M}_{i j}+\frac{1}{2} \sum_{i=1}^{r} \sum_{j=1}^{n} \sum_{k=1}^{n} \frac{\partial \mathrm{F}_{i j}}{\partial \mathrm{M}_{i j}} \delta \mathrm{M}_{i j} \delta \mathrm{M}_{i k}>0
$$

for all the virtual changes. If this inequality is satisfied and if all the virtual changes are revertible, we may also write the following inequality:

$$
-\sum_{i=1}^{r} \sum_{j=1}^{n} \mathrm{~F}_{i j} \delta \mathrm{M}_{i j}+\frac{1}{2} \sum_{i=1}^{r} \sum_{j=1}^{n} \sum_{k=1}^{n} \frac{\partial \mathrm{F}_{i j}}{\partial \mathrm{M}_{i k}} \delta \mathrm{M}_{i j} \delta \mathrm{M}_{i k}>0 .
$$

From these two inequalities it follows that we must have:

$$
\begin{gathered}
\sum_{i=\mathrm{I}}^{r} \sum_{j=\mathrm{I}}^{n} \mathrm{~F}_{i j} \delta \mathrm{M}_{i j}=0, \\
\sum_{i=\mathrm{I}}^{r} \sum_{j=\mathrm{I}}^{n} \sum_{k=\mathrm{I}}^{n} \frac{\partial \mathrm{F}_{i j}}{\partial \mathrm{M}_{i k}} \delta \mathrm{M}_{i j} \delta \mathrm{M}_{i k}>0 .
\end{gathered}
$$

Accordingly the system will be in stable equilibrium if the conditions 95 and 96 are satisfied. Equation 95 is the wellknown equation from which the conditions of equilibrium of the system are obtained, that is to say the various theorems which constitute the phase rule; the inequality 96 will enable us to establish the theorems which are consequences of the stability of the equilibrium. 
If the system admits virtual changes such that

$$
\frac{\delta \mathrm{M}_{i \mathrm{I}}}{\mathrm{M}_{i \mathrm{I}}}=\frac{\delta \mathrm{M}_{i 2}}{\mathrm{M}_{i 2}}=\ldots=\frac{\delta \mathrm{M}_{i n}}{\mathrm{M}_{i n}}, \quad i=\mathrm{I}, 2, \ldots, r,
$$

we sha11 have for those changes:

$$
\sum_{i=1}^{r} \sum_{j=1}^{n} \sum_{k=1}^{n} \frac{\partial F_{i j}}{\partial M_{i k}} \delta M_{i j} \delta M_{i k}=0
$$

To show this we observe that in this equation the coefficient of $\delta \mathrm{M}_{i k}$ can be written in the following ways:

$$
\sum_{j=1}^{n} \frac{\partial \mathrm{F}_{i j}}{\partial \mathrm{M}_{i k}} \delta \mathrm{M}_{i j}=\sum_{j=\mathrm{I}}^{n} \frac{\partial^{2} \Phi_{i}}{\partial \mathrm{M}_{i j} \partial \mathrm{M}_{i k}} \delta \mathrm{M}_{i j}=\sum_{j=1}^{n} \frac{\partial \mathrm{F}_{i z}}{\partial \mathrm{M}_{i j}} \delta \mathrm{M}_{i j}
$$

If the equations 97 are satisfied the last of these expressions is proportional to

$$
\sum_{j=\mathrm{I}}^{n} \frac{\partial F_{i k}}{\partial \mathrm{M}_{i j}} \mathrm{M}_{i j}
$$

and as $F_{i k}$ is a homogeneous function of order zero, this expression is equal to zero.

In the discussion which follows we shall only consider systems whose equilibrium is such that

$$
\sum_{i=1}^{r} \sum_{j=1}^{n} \sum_{k=1}^{n} \frac{\partial \mathrm{F}_{i j}}{\partial \mathrm{M}_{i k}} \mathrm{X}_{i j} \mathrm{X}_{i \bar{i}}>0
$$

according as the equations

$$
\frac{\mathrm{X}_{i \mathrm{1}}}{\mathrm{M}_{i \mathrm{I}}}=\frac{\mathrm{X}_{i 2}}{\mathrm{~N}_{i 2}}=\ldots=\frac{\mathrm{X}_{i n}}{\mathrm{M}_{i n}}, \quad i=\mathrm{I}, 2, \ldots, r,
$$

are or are not satisfied. These systems will accordingly be in stable or in indifferent equilibrium.

By a demonstration analogous to the one we have just given we can show that

$$
\sum_{i=1}^{r} \sum_{j=1}^{n} \sum_{k=1}^{n} \frac{\partial \mathrm{F}_{i j}}{\partial \mathrm{M}_{i k}} \mathrm{X}_{i j} \mathrm{Y}_{i k}=0
$$


when

$$
\frac{\mathrm{X}_{i \mathrm{I}}}{\mathrm{M}_{i \mathrm{I}}}=\frac{\mathrm{X}_{i 2}}{\mathrm{I}_{i 2}}=\ldots=\frac{\mathrm{X}_{i n}}{\mathrm{M}_{i n}}, \quad i=\mathrm{I}, 2, \ldots, r, \quad \text { (IOI) }
$$

or when

$$
\frac{\mathrm{Y}_{i \mathrm{I}}}{\mathrm{M}_{i \mathrm{I}}}=\frac{\mathrm{Y}_{i 2}}{\mathrm{M}_{i 2}}=\ldots=\frac{\mathrm{Y}_{i n}}{\mathrm{M}_{i n}}, \quad i=\mathrm{I}, 2, \ldots, r . \quad \text { (IO2) }
$$

Let us now return to the consideration of a system in equilibrium, at a temperature $T$, under a pressure $I I$ and for certain values of the masses $\mathrm{M}_{i j}$. From condition 95 we shall have

$$
\sum_{i=\mathrm{I}}^{r} \sum_{j=\mathrm{I}}^{n} \mathrm{~F}_{i j} \delta \mathrm{M}_{i j}=0
$$

for every virtual change which leaves the temperature, the pressure and the mass of the system unchanged. To express that the system is in equilibrium in a state adjacent to the first state of equilibrium, we must replace $T, \Pi, M_{i j}$ in the preceding equation by $\mathrm{T}+d \mathrm{~T}, \Pi \leq d \Pi, \mathrm{M}_{i j} \div d \mathrm{M}_{i j}$. We thus obtain :

$$
\begin{aligned}
d \Pi \sum_{i=1}^{r} \sum_{j=1}^{n} \frac{\partial \mathrm{F}_{i j}}{\partial \Pi} \delta \mathrm{M}_{i j} & +d^{\top} \mathrm{T} \sum_{i=\mathrm{I}}^{r} \sum_{j=\mathrm{I}}^{n} \frac{\partial \mathrm{F}_{i j}}{\partial \mathrm{T}} \delta \mathrm{M}_{i j} \\
& +\sum_{i=\mathrm{I}}^{r} \sum_{j=\mathrm{I}}^{n} \sum_{k=\mathrm{I}}^{n} \frac{\partial \mathrm{F}_{i j}}{\partial \mathrm{M}_{i k}} d \mathrm{M}_{i k} \delta \mathrm{M}_{i j}=\mathrm{o} . \quad \text { (IO3) }
\end{aligned}
$$

Now, when the system is in equilibrium, the volume $V$ and the entropy $\mathrm{H}$ of the system are given by the formulas

$$
\begin{array}{r}
V=\frac{\partial \Phi}{\partial \Pi}=\sum_{i=1}^{r} \sum_{j=\mathrm{I}}^{n} \frac{\partial \mathrm{F}_{i j}}{\partial \Pi} \mathrm{M}_{i j} . \\
-\mathrm{H}=\frac{\partial \Phi}{\partial \mathrm{T}}=\sum_{i=\mathrm{I}}^{r} \sum_{j=\mathrm{I}}^{n} \frac{\partial \mathrm{F}_{i j}}{\partial \mathrm{T}} \mathrm{M}_{i j} .
\end{array}
$$


As $\mathrm{F}_{i j}$ is a homogeneous function of the masses $\mathrm{M}_{i \mathrm{1}}, \mathrm{M}_{i 2}, \ldots$, $M_{i n}$, of degree zero, its derivatives with respect to $\Pi$ and to $T$ are also homogeneous functions of degree zero. It follows then without difficulty, that, for a virtual change which leaves the temperature and the pressure unchanged, the variations of the volume and of the entropy are given by the formulas:

$$
\begin{array}{r}
\delta \mathrm{V}=\sum_{i=1}^{r} \sum_{j=\mathrm{I}}^{n} \frac{\partial \mathrm{F}_{i j}}{\partial \mathrm{II}} \delta \mathrm{M}_{i j}, \\
-\delta \mathrm{H}=\sum_{i=\mathrm{I}}^{r} \sum_{j=\mathrm{I}}^{n} \frac{\partial \mathrm{F}_{i j}}{\partial \mathrm{T}} \delta \mathrm{M}_{i j} .
\end{array}
$$

By means of these formulas we can write equation IO3 in the form

$$
d \mathrm{II} \cdot \delta \mathrm{V}-d \mathrm{~T} \cdot \delta \mathrm{H}+\sum_{i=1}^{r} \sum_{j=\mathrm{I}}^{n} \sum_{k=\mathrm{I}}^{n} \frac{\partial \mathrm{F}_{i j}}{\partial \mathrm{M}_{i k}} d \mathrm{M}_{i j} \delta \mathrm{M}_{i j}=0 . \quad \text { (108) }
$$

From this equation, which is due to Planck, ${ }^{2}$ we shall now derive the various theorems relating to the displacement of equilibrium.

Consider first an invariant system. As in this case the temperature and the pressure are fixed we have

$$
d \Pi=0, \quad d \mathrm{~T}=\mathrm{o} .
$$

The masses of the phases may vary but the concentrations must remain unchanged; accordingly we have

$$
\frac{d \mathrm{M}_{i \mathrm{I}}}{\mathrm{M}_{i \mathrm{I}}}==\frac{d \mathrm{M}_{i 2}}{\mathrm{M}_{i 2}}=\ldots=\frac{d \mathrm{M}_{i n}}{\mathrm{M}_{2 n}}, \quad i=\mathrm{I}, 2, \ldots, r
$$

Equations roo and IoI show us that the last term of equation I08 reduces to zero. In the case of invariant systems each term of equation 108 is thus equal to zero.

Consider now a univariant system. In such a system, at a given temperature, the pressure and the concentrations are determined, but the masses of the phases may vary. We can thus

\footnotetext{
1 Vorlesungen über Thermodynamik, p. I76.
} 
choose a virtual change which will leave the concentrations n11changed; that is to say we may write

$$
\frac{\delta \mathrm{M}_{i \mathrm{I}}}{\mathrm{M}_{i \mathrm{I}}}=\frac{\delta \mathrm{M}_{i_{2}}}{\mathrm{M}_{i 2}}=\ldots=\frac{\delta \mathrm{M}_{i n}}{\mathrm{M}_{i n}} .
$$

The third term of equation 108 is then equal to zero and that equation reduces to

$$
d \Pi \cdot \delta \mathrm{V}-d^{\top} \mathrm{T} \cdot \delta \mathrm{H}=\mathrm{o}
$$

or if we write

we get

$$
Q=\mathrm{T} \cdot \delta \mathrm{H}
$$

$$
\frac{d \Pi}{d 1}=\frac{Q}{T \delta V} .
$$

In this equation $Q$ denotes the quantity of heat absorbed by the systen and $\delta \mathrm{V}$ the increase in volume when, at constant temperature and pressure, the system undergoes a change which leaves the concentrations unchanged. The above demonstration of Clapeyron's equation is due to Planck. ${ }^{\mathrm{r}}$

Last consider a bivariant or multivariant system. In such a system, if the temperature and the pressure be given, the state of the system is completely determined; it is impossible to choose a virtual change which shall leave the concentrations unchanged. Let us take as a virtual change the real change that takes place when the system passes from one state of equilibrium to the next; that is to say, let us take

$$
\delta \mathrm{M}_{i j}=d \mathrm{M}_{i j} \text {. }
$$

Inequality 98 shows us that the third term in equation 108 is positive; that equation accordingly takes the form

$$
d \Pi \cdot \delta \mathrm{V}-d \mathrm{~T} \cdot \delta \mathrm{H}<\mathrm{o}
$$

or, if we write

we get

$$
\mathrm{Q}=\mathrm{T} \delta \mathrm{H}
$$

$$
d \mathrm{II} \cdot \delta \mathrm{V}-d \mathrm{~T} \frac{\mathrm{Q}}{\mathrm{T}}<\mathrm{O} .
$$

1 Vorlesungen über Thermodynamik, p. 176. 
In this inequality $\delta \mathrm{V}$ is the change which the volume of the system would undergo and $Q$ the quantity of heat which the system would absorb if the changes which really take place in the concentrations when the system passes from one state of equilibrium to the next, were to take place at constant temperature and pressure.

From the inequality I Io we get the two following results:

$$
\begin{array}{ll}
d \mathrm{~T}=0, & d \Pi \cdot \delta \mathrm{V}<0 ; \\
d \Pi=0, & d \mathrm{~T} \cdot \mathrm{Q}>0 .
\end{array}
$$

The first of these results gives us Le Châtelier's theorem: ${ }^{r}$ If, at a constant temperature, the pressure of a bivariant or nultivariant system in equilibrium be increased, there takes place a change in the concentrations which would have for effect a diminution of the volume of the system if the change were to take place at constant temperature and pressure. Similarly a diminution of pressure would cause a change corresponding to an increase of volume.

The second of the preceding results gives us van't Hoff's theorem: ${ }^{2}$ If, under constant pressure the temperature of a bivariant or multivariant system in equilibrium be raised, there takes place a change in the concentrations which would have for effect an absorption of heat if the change were to take place at constant temperature and pressure. Similarly, a lowering of the temperature would cause a change corresponding to the liberation of heat.

In the particular case of a bivariant or multivariant system at an indifferent point, we can vary the masses of the phases without changing the concentrations, that is to say we may take

$$
\frac{\delta \mathrm{M}_{i \mathrm{1}}}{\mathrm{M}_{i \mathrm{1}}}=\frac{\delta \mathrm{M}_{i 2}}{\mathrm{M}_{i 2}}:=\cdots=\frac{\delta \mathrm{M}_{i n}}{\mathrm{M}_{i n}}, \quad i=\mathrm{I}, 2, \ldots, r .
$$

${ }^{1}$ Compt. rend. 99, 786 (1884). Duhem. Annales de la Faculté de Toulouse, 4, N; ( I890). Traité élementaire de Mécanique chimiqne, I, I45.

${ }^{2}$ Études de Dynamique chimique, I6I; Amsterdam, 1884. Duhem. Annales de la Faculté de Toulouse, 4, N; (1890). Traité élementaire de Mécanique chimique, I, 184. 
The third term of equation 108 then reduces to zero and that equation becomes

$$
d \Pi \cdot \delta \mathrm{V}-d \mathrm{~T} \cdot \delta \mathrm{H}=\mathrm{o}
$$

or if we put

$$
Q=T \delta H
$$

we get

$$
\frac{d \Pi}{d T}=\frac{Q}{T \delta V}
$$

In this equation $\delta \mathrm{V}$ is the increase which the volume of the system undergoes and $Q$ the quantity of heat absorbed by the system, supposed to be at an indifferent point, when the masses of the phases are varied without changing the temperature, the pressure or the concentrations. We have already met this extension of Clapeyron's formula.

Consider again a bivariant or a multivariant system. If the system undergoes a reversible isothermal change, we have constantly $d^{\prime} \mathrm{T}=0$. When the system reaches an indifferent point we can choose the variations $\delta \mathrm{M}_{i j}$, so that equations II 3 are satisfied. Equation Io8 then becomes

$$
d \Pi \cdot \delta \mathrm{V}=0
$$

in which $\delta \mathrm{V}$ is the change which the volume of the system undergoes at the indifferent point when we vary the masses of the phases, but keep the temperature, pressure and concentrations constant. If $\delta \mathrm{V}$ is not equal to zero, we must have

$$
d \Pi=\mathrm{o} .
$$

We thus obtain the first of Gibbs' theorems : ${ }^{I}$ At an indifferent point of a bivariant or multivariant system kept at a constant temperature, the pressure is, in general, a maximum or a minimum.

Similarly, if we consider a reversible change, accomplished under constant pressure, we see that at an indifferent point

$$
d \mathrm{~T} \cdot \delta \mathrm{H}=\mathrm{o},
$$

${ }^{1}$ On the Equilibrium of Heterogeneous Substances, p. I55. Konowalow. Wied. Ann. 14, 48 (188I). 
or if we put

$$
\begin{array}{r}
\mathrm{Q}=\mathrm{T} \delta \mathrm{H}, \\
d \mathrm{~T} \cdot \mathrm{Q}=\mathrm{o} .
\end{array}
$$

$Q$ denotes the quantity of heat which the system absorbs when, under a constant pressure and at the temperature of the corresponding indifferent point, the system undergoes a change in which the masses of the phases vary while the concentrations remain constant. If we suppose that $Q$ is not equal to zero, we must have

$$
d \mathrm{~T}=\mathrm{o} .
$$

We thus obtain the second of Gibbs' theorems: ${ }^{x}$ At an indifferent point of a bivariant or multivariant system kept under constant pressure, the temperature is, in general, a maximum or a minimum.

We can obtain an interesting result by comparing equations rog and II4. For that purpose let us consider a univariant system and the bivariant system which we obtain by removing one of the phases of the nnivariant system. If, in a $\Pi, T$ - plane, we draw the curve which represents the temperatures and the equilibrium pressures of the univariant system and also the curve which represents the temperatures and the pressures of the indifferent points of the bivariant system, the comparison of equations IOg and II 4 shows us that the two curves are tangent to each other at their point or points of intersection.

Suppose, for example, that the univariant system consists of the three phases : a solid hydrate, a solution of the hydrate in water and a vapor phase, and that the corresponding bivariant system consists of the two phases: solid hydrate and solution. If we notice that in the bivariant system the change in volume which occurs when a portion of the hydrate enters into solution is small in comparison with the quantity of heat absorbed during the same process, we see that the curve of indifferent points is very nearly perpendicular to the axis of temperatures. Accordingly, in the univariant system, when the hydrate and the

On the Equilibrium of Heterogeneous Substances, p. I55. 
solution have the same concentration, the tangent to the equilibrium curve is very nearly perpendicular to the axis of temperatures; it follows that at that point the temperature passes, in general, through a maximum or a minimum. This theorem is due to van der Waals, ${ }^{\mathrm{x}}$

We can derive another particular result from the general equation 22. Suppose that the system under sonsideration is a system of two phases formed by means of two components, and that the concentration of one of the phases is necessarily constant. As examples we may mention the system: salt solution and water vapor and the system: solid salt and solution. Suppose that the variable concentration remains constant, then we shall have

$$
\frac{d \mathrm{M}_{i 1}}{\mathrm{M}_{i \mathrm{I}}}=\frac{d \mathrm{M}_{i 2}}{\mathrm{M}_{i 2}}, \quad i=\mathrm{I}, 2,
$$

and equation 108 will take the form

$$
\frac{d \Pi \mathrm{II}}{d \mathrm{~T}}=\frac{\mathrm{Q}}{\mathrm{T} \delta \mathrm{V}} .
$$

In this formula $\delta \mathrm{V}$ represents the change which the volume of the system undergoes and $Q$ the quantity of heat which the system absorbs during a virtual change accomplished at constant temperature and pressure. This equation has been given by Braun $^{2}$ and Natanson. ${ }^{3}$

${ }^{1}$ Bakhius Roozeboom. Sur les conditions d'équilibre de deux corps dans les trois états, solide, liquide et gazeux, d'après M. v. d. Waals. Recueil des Travaux chimiques des Pays-Bas, 5, 335 ( 1886). Zeit. phys. Chem, 2, 463 (I888). Duhem. Dissolutions et Mélanges, deuxième Mémoire: les Propriétés physiques des Dissolutions; Travaux et Mémoires des Facultés de Lille, 3, No. I2, I2I, ( 1893 ). Traité élémentaire de Mécanique chimique, 3, 279.

2Wied. Ann. 30, 250 (1887). Duhem. Dissolutions et Mélanges deuxième Mémoire: les Propriétés physiques des Dissolutions; Travaux et Mémoires des Facultés de Lille, 2, No. I2, 29 (I893). Traité élémentaire de Mécanique chimique, 3, r 30 .

${ }^{3}$ Zeit. phys. Chem. I0, 748 ( I 892 ). Duhem. Travaux et Mémoires des Facultés de I,ille, 2, No, I2, 72 (1893). Traite élémentaire de Mécanique chimique, 3,156 . 